\title{
GNSS Carrier Phase Ambiguity Resolution: Challenges and Open Problems
}

\author{
P.J.G. Teunissen and S. Verhagen
}

\begin{abstract}
Integer carrier phase ambiguity resolution is the key to fast and high-precision global navigation satellite system (GNSS) positioning and application. Although considerable progress has been made over the years in developing a proper theory for ambiguity resolution, the necessary theory is far from complete.

In this contribution we address three topics for which further developments are needed. They are: (1) Ambiguity acceptance testing; (2) Ambiguity subset selection; and (3) Integer-based GNSS model validation. We will address the shortcommings of the present theory and practices, and discuss directions for possible solutions.
\end{abstract}

Keywords Ambiguity acceptance tests - Ambiguity subset selection. Integer based GNSS model validation

\section{Introduction}

Integer carrier phase ambiguity resolution is the key to fast and high-precision GNSS positioning and navigation. It is the process of resolving the unknown cycle ambiguities of the double-differenced carrier phase data as integers. Once this has been done successfully,

P.J.G. Teunissen

Delft Institute of Earth Observation and Space systems, Delft University of Technology, Kluyverweg 1, 2629 HS Delft, The Netherlands, e-

mail: P.J.G.Teunissen@TUDelft.nl/A.A.Verhagen@TUDelft.nl

S. Verhagen

Delft Institute of Earth Observation and Space systems, Delft University of Technology, Kluyverweg 1, 2629 HS Delft, The Netherlands the very precise carrier phase data will act as pseudo range data, thus making very precise positioning and navigation possible.

GNSS ambiguity resolution (AR) applies to a great variety of current and future models of GPS, modernized GPS and Galileo, with applications in surveying, navigation, geodesy and geophysics. These models may differ greatly in complexity and diversity. They range from single-baseline models used for kinematic positioning to multi-baseline models used as a tool for studying geodynamic phenomena. The models may or may not have the relative receiver-satellite geometry included. They may also be discriminated as to whether the slave receiver(s) is stationary or in motion, or whether or not the differential atmospheric delays (ionosphere and troposphere) are included as unknowns. An overview of these models can be found in textbooks like (Strang and Borre, 1997; Teunissen and Kleusberg, 1998; Hofmann-Wellenhoff et al., 2001; Leick, 2003; Misra and Enge, 2001).

Despite the key role that is played by GNSS AR in the various high-precision applications, the necessary theory is far from completed. Consider the conceptual steps that can be recognised in GNSS AR:

1. In the first step, one discards the integer nature of the ambiguities and performs a standard least-squares adjustment. As a result one obtains the so-called float solution of all the parameters (i.e. ambiguities, baseline components, and possibly additional parameters such as atmospheric delays), together with their variance-covariance matrix. In this first step, one usually also tests the data and the assumed GNSS model for possible model misspecifications, e.g. outliers, cycle slips, or other modeling errors. This can be done with the 
standard theory of hypothesis testing, (Koch, 1999; Teunissen, 2006).

2. In the second step, the real-valued float solution of the ambiguities is further adjusted, so as to take the integer constraints into account. As a result one obtains an integer solution for the ambiguities. The integer estimators mostly used in practice are:

- Integer least squares (ILS): ILS is the optimal method for integer estimation as it can be shown to maximize the probability of correct integer estimation (Teunissen et al., 1999). ILS requires a search which can be done very efficiently with the LAMBDA method. The evaluation of the ILS success rate requires simulation, (Teunissen, 1998a; Verhagen, 2005a; Joosten and Tiberius, 2000), the use of adequate approximations such as based on the ADOP (Teunissen, 1997a; Ji et al., 2007; Lee et al., 2005; Vollath et al., 2003), or the use of upper- and lower bounds, (Teunissen, 1998a; Verhagen, 2005a).

- Integer Bootstrapping (IB): The advantage of IB over ILS is that no search is needed. Although suboptimal, the performance of IB can come close to that of ILS if the appropriate decorrelating $Z$-transformation is used. An advantage of IB is that its success rate can be computed very easily, (Teunissen, 1998b).

- Integer Rounding (IR): This method is the simplest of all, but it also has the lowest success rate of all. Still, it can be a useful method if the strength of the underlying GNSS model is such that the IR success rate is large. Often, as in case with IB, this first requires the application of a decorrelating $Z$-transformation.

3. Once the integer ambiguities are computed, they are used in the third step as input to decide whether or not to accept the integer solution. Several such tests have been proposed in the literature and are currently in use in practice, (Abidin, 1993; Chen, 1997). Examples are the ratio-test, the distance-test and the projector-test. A review and evaluation of these tests can be found in (Verhagen, 2005b).

4. Knowing that the ambiguities are integer, strengthens the model and allows one, in principle, to reevaluate the validation of the GNSS model. In practice this is done by applying the standard theory of hypothesis testing, thereby assuming the ambiguities as known.

5. Once the GNSS model and integer solution are accepted, the last step consists of correcting the float solution of all other parameters by virtue of their correlation with the ambiguities. As a result one obtains the so-called fixed solution. Provided a correct decision has been made in the third and fourth step, the fixed solution will have a precision that is in accordance with the high precision of the phase data.

In this contribution we address and discuss three topics for which the theory and/or numerical implementation need further improvements. The three topics are: (1) Ambiguity Acceptance Tests: one of the most popular such test is the ratio-test. We will discuss its relevance and performance, and show that its current usage is not optimal. (2) Ambiguity Subset Selection: since full AR may not always be possible, partial AR provides an alternative. We will discuss some of the issues of partial AR. (3) Integer-Based Model Validation: we will argue that the standard theory of linear model hypothesis testing is not quite applicable to this case. A first approach to perform integer-based model validation will be given.

\section{Ambiguity Acceptance Tests}

\subsection{Ratio-Test}

One of the most popular ambiguity acceptance tests is the so-called ratio-test. The ratio-test is defined as follows. Let the float ambiguity vector and its variance matrix be given as $\hat{a}$ and $Q_{\hat{a} \hat{a}}$, respectively. Furthermore, let $\hat{a}$ be the ILS solution, i.e. the integer minimizer of $q(a)=(\hat{a}-a)^{T} Q_{\hat{a} \hat{a}}^{-1}(\hat{a}-a)$, and let $\check{a}^{\prime}$ be the integer vector that returns the second smallest value of the quadratic form $q(a)$. Then the ratio-test reads as:

$$
\text { Accept } \check{a} \text { iff }: \frac{q\left(\check{a}^{\prime}\right)}{q(\check{a})} \geq c
$$

where $c$ is a tolerance value, to be selected by the user. Thus only if $q\left(\check{a}^{\prime}\right)$ is sufficiently larger than $q\left(\check{a}^{\prime}\right)$, will the decision be made to accept the ILS solution. Otherwise, the ILS solution is rejected in favor of the float solution. 
Questions that need to be addressed when using the above test are: (1) What does the ratio-test actually test? (2) What errors can be made with the ratio-test? (3) What value for $c$ should be chosen? Answers to these questions are needed, in order to have a proper understanding of the ratio-test.

Let us first see how the test is executed in practice. With reference to the theory of hypothesis testing, the ratio of the two quadratic forms, $q(\check{a})$ and $q\left(\check{a}^{\prime}\right)$, is often assumed to have a Fisher-distribution, from which $c$ can be computed, once the level of significance has been set. The problem with this approach is, however, that the ratio of the two quadratic forms is not Fisherdistributed. Even if one was allowed to assume that $q(\check{a})$ and $q\left(\check{a}^{\prime}\right)$ are Chi-square distributed (which is not true, since also the uncertainty of the integer vectors needs to be taken into account), then their ratio would still not be Fisher-distributed. The two quadratic forms are namely not independent.

Also, in many of the existing software packages, a fixed value for $c$ is chosen, no matter the varying strength of the underlying GNSS model. Examples of proposed $c$-values are given in e.g. (Wei and Schwarz, 1995; Han and Rizos, 1996; Euler and Schaffrin, 1991). It is however strange to base the testing on a fixed $c$-value, since one would expect that with a varying strength of the GNSS model, the use of different measurement scenarios or with varying degrees of freedom, one also would use varying values for $c$. The incorrect assumption of the distributional properties of the ratio-test and the often used fixed $c$-value approach, can be explained by the lack of a proper theory (Teunissen and Verhagen, 2007b).

What does the ratio-test actually test? One motivation that is often given for the use of the ratio-test, is that it tests the correctness of the ILS solution. This is, however, incorrect, since one can add an arbitrary integer vector to the float solution, without altering the outcome of the ratio-test. Hence, biases of arbitrary size (provided they are integer) can be present in the float solution, without them ever being noticed by the ratio-test.

\subsection{Relevance of the Ratio-Test}

To understand what the ratio-test tests, we need to get a better insight into its acceptance region and rejection region. We already remarked that the outcome of the ratio-test remains unchanged when an arbitrary integer vector, say $z$, is added to the float solution. This implies that its acceptance region, denoted as $\Omega$, must be a region which is $z$-translational invariant. That is, if the acceptance region is translated over an arbitrary integer vector, then the same acceptance region is recovered again. Since the rejection region is complementary to the acceptance region, also the rejection region of the ratio-test is $z$-translational invariant.

The $z$-translational invariance of the acceptance region, implies that it must equal the union of $z$-translated copies of a smaller region $\Omega_{0}$. Thus

$$
\Omega=\bigcup_{z \in \mathbb{Z}^{n}} \Omega_{z} \text { where } \Omega_{z}=\Omega_{0}+z
$$

The region $\Omega_{z}$ is called the aperture pull-in region of the integer $z$ (Teunissen, 2003). The aperture pull-in regions $\Omega_{z}$ of the ratio-test are given in (Teunissen and Verhagen, 2004; Verhagen and Teunissen, 2006). For the test, we have

$$
\hat{a}=z \text { if } \hat{a} \in \Omega_{z}
$$

Thus if the float solution resides in $\Omega_{z}$, the ratio-test leads to acceptance and the ILS solution is equal to $z$. Hence, the ratio-test does not test the correctness of the ILS-solution. Instead, the ratio-test tests the closeness of the float solution to its nearest integer vector. If it is close enough, the test leads to acceptance of $\hat{a}$. If it is not close enough, then the test leads to rejection in favor of the float solution $\hat{a}$. The size or aperture of the pull-in region provides the largest distance one is willing to accept. The value for $c$ can be used to tune this aperture.

To understand in what way the ratio-test helps us in getting confidence in the outcome of ambiguity resolution, we have to realize that acceptance of the ILS solution by the ratio-test can be correct or incorrect. We therefore have to distinguish between the following three cases:

$$
\begin{array}{ll}
\hat{a} \in \Omega_{a} & \text { success: correct integer estimation } \\
\hat{a} \in \Omega\left\{\Omega_{a}\right\} & \text { failure: incorrect integer estimation } \\
\hat{a} \notin \Omega & \text { undecided: ambiguity not fixed to an } \\
& \text { integer }
\end{array}
$$

where $\Omega \backslash \Omega_{a}$ means that $\Omega_{a}$ is deleted from the set $\Omega$, with $a$ being the unknown integer ambiguity vector. 
The corresponding probabilities are denoted as: $P_{S}$ (success-rate), $P_{f}$ (failure-rate) and $P_{u}$ (probability of undecidedness). The probability $P_{s}+P_{f}$ is the probability of acceptance of the ratio-test and $P_{u}$ is its probability of rejection. The probability of having successful fixes, denoted as $P_{s f}$, is given by the ratio of the success rate and the probability of acceptance,

$$
P_{s f}=\frac{P_{s}}{P_{s}+P_{f}}
$$

The above probabilities all depend on the shape and size of $\Omega_{0}$ and on the PDF of $\hat{a}$. Thus by changing $\Omega_{0}$ and/or the PDF of $\hat{a}$, one can influence the above probabilities. Changing the PDF will not be possible, once the measurement scenario is given (this will be different in case one is designing a measurement scenario). Changing the shape of $\Omega_{0}$ is also not possible, since the shape is determined by the ratio-test. This leaves us with the size of $\Omega_{0}$, which is determined by $c$. Hence, by changing $c$ one can influence the above probabilities. Thus through the choice of $c$, the user is able to have control over the failure rate and the probability of successful fixing. This is important, because it gives the user the necessary flexibility over what he/she finds an acceptable risk to take with integer ambiguity resolution. This is the relevance of having the ratio-test included in GNSS AR.

The above discussion makes clear that the common practice of using a fixed value for $c$ is therefore not the way to go. By using a fixed value for $c$, the user is deprived from any control over the failure rate. The failure rate will then be different for different measurement scenarios. Already in a kinematic or navigation scenario where data are collected on an epoch by epoch basis, the failure rate can change from epoch to epoch if a fixed value for $c$ is used.

To use the fixed failure-rate approach, one has to be able to compute $c$ from the failure-rate $P_{f}$ as set by the user. This is a nontrivial numerical task for which a practical approach has been devised in (Verhagen, 2005b; Teunissen and Verhagen, 2007a). A challenge remains however to make this approach numerically efficient for real-time applications.

\subsection{Is the Ratio-Test Optimal?}

The answer is no, even if the ratio-test would be used with a fixed failure rate instead of with a fixed value for $c$. It can be shown that the ratio-test is a member of the class of tests as given by the theory of integer aperture estimation developed by (Teunissen, 2003). Members from this class differ in the way the shape of the aperture pull-in region $\Omega_{0}$ is defined. Hence, within this class, one can, by fixing the failure rate, solve for the aperture pull-in region that maximizes the success rate (Fig. 1 gives a two-dimensional example of the optimal aperture pull-in regions). The optimal test so obtained differs from the ratio-test. For a discussion of the optimal test and its relation to the ratio-test, we refer to (Teunissen, 2003; Verhagen and Teunissen, 2006). As with the ratio-test, efficient computation of the tolerance value from the user-defined failure-rate remains a challenge.

\section{Ambiguity Subset Selection}

\subsection{Motivation}

There are two reasons why we address the topic of ambiguity subset selection. The first reason has to do with ones ability to resolve all ambiguities. Full AR, i.e. the successful resolution of all ambiguities, may not always be possible. This will occur when the underlying GNSS model lacks sufficient strength. When one aims at resolving all, say $n$, ambiguities, one requires that the simultaneous event $\bigcap_{i=1}^{n}\left\{\check{a}_{i}=a_{i}\right\}$ has a probability close to 1 . This probability has the general tendency, however, to get smaller as $n$ gets larger. One can speak of a dimensional curse. A way out of this dilemma, when it occurs, is to aim at resolving only an ambiguity subset, thus keeping the dimension bounded.

The second reason has to do with the necessity of full AR. Full AR may, in fact, not always be needed. As an extreme example consider the case where the float ambiguities are not correlated with the other float parameters. Ambiguity resolution would then be useless, since it will then not allow for an improvement of these other parameters. This indicates that resolving a subset of ambiguities (in the extreme example, an empty subset) may lead to the same, or almost same, performance improvement of the other parameters. The observation that the resolution of different ambiguity subsets, has different impacts on the performance 
Fig. 1 Two-dimensional example of optimal aperture pull-in regions (gray areas), together with the ILS pull-in regions (hexagons)

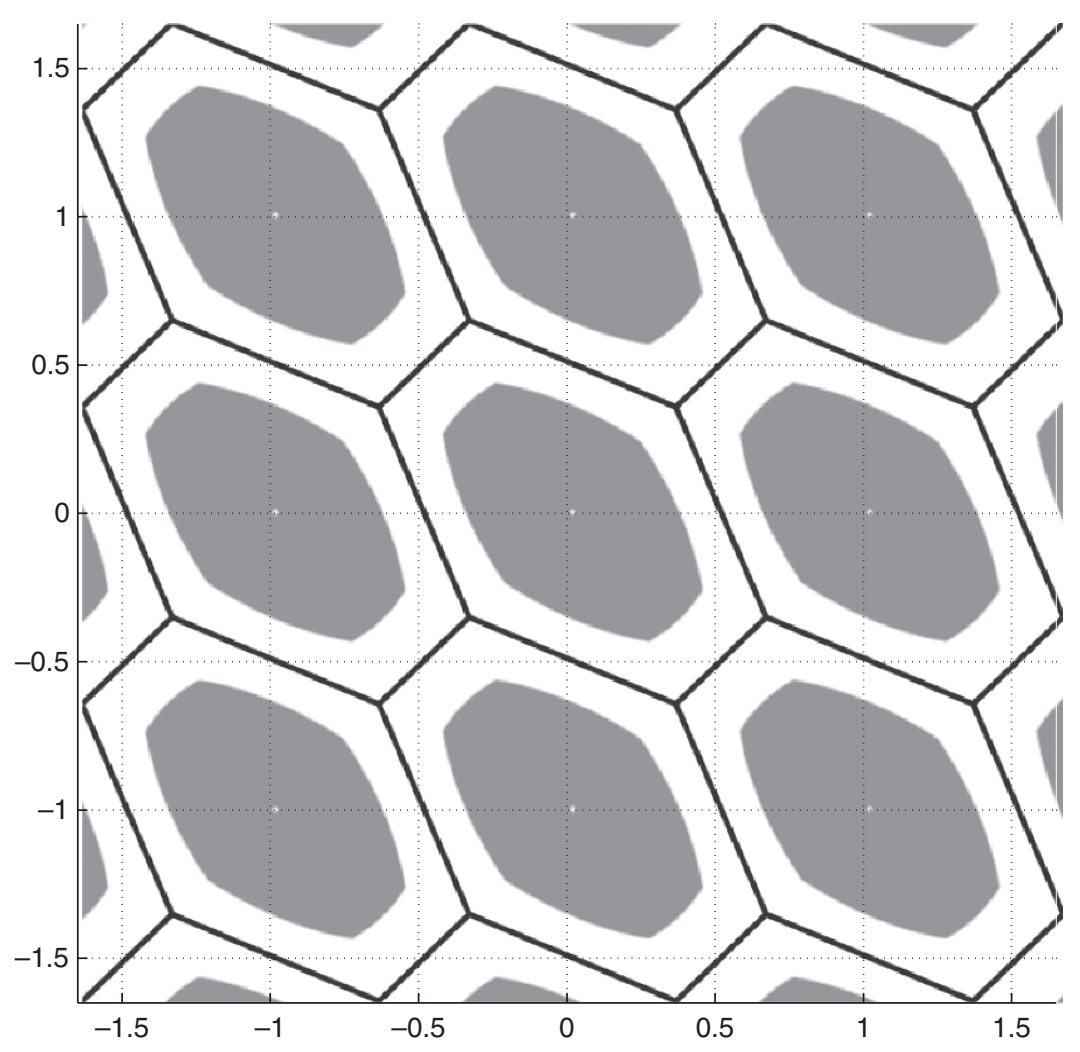

improvements, brings the question to the fore which subset to choice. This problem of identifying the optimal ambiguity subset, in relation to the performance improvement it brings, has not yet been addressed in the literature.

\subsection{Partial Ambiguity Resolution}

Let us now continue with the case for which it turns out that full AR is impossible (i.e. the success rate is too low). Partial AR may then be a good alternative. This idea of partial ambiguity resolution was introduced in (Teunissen et al., 1999), where it was applied to long baselines using the current GPS, see also the review (Teunissen, 2001). The goal of partial AR is to identify the ambiguity subset which gives the largest possible success rate. Partial AR based on integer bootstrapping goes as follows. It will be clear that the subset selection should be based on the precision of the 'float' ambiguities. The more precise the ambiguities, the larger the ambiguity success rate. Therefore, first the decorrelation step of the LAMBDA method is used, followed by an application of the bootstrapping principle. Once the transformed and decorrelated ambiguity variance matrix is obtained, the construction of the subset proceeds in a sequential fashion. One first starts with the most precise ambiguity, say $\hat{z}_{1}$, and computes its success rate $P\left(\check{z}_{1}=z_{1}\right)$. If this success rate is large enough, one continues and determines the most precise pair of ambiguities, say $\left(\hat{z}_{1}, \hat{z}_{2}\right)$. If their success rate is still large enough, one continues again by trying to extend the set. This procedure continues until one reaches a point where the corresponding success rate becomes unacceptably small. When this point is reached, one can expect that the previously identified ambiguities can be resolved successfully.

This method of ambiguity subset selection has been shown to work very well in practice. The method is applicable to any GNSS model and it is superior to 'widelaning' methods (Teunissen, 1997b; Teunissen et al., 1999). However, the identified problem of optimal ambiguity subset selection has not yet been completely solved with the above approach. The above approach, which is based on bootstrapping, identifies the optimal subset for sequential AR and not necessarily for batch AR. This latter problem is not yet solved. 


\section{Integer-Based GNSS Model Validation}

\subsection{Problem Statement}

Consider the following null- and alternative hypothesis

$$
\begin{array}{r}
H_{0}: y \sim \mathrm{N}\left(A a+B b, Q_{y y}\right), a \in \mathbb{Z}^{n}, b \in \mathbb{R}^{p} \\
H_{a}: y \sim \mathrm{N}\left(A a+B b+C c, Q_{y y}\right), a \in \mathbb{Z}^{n}, \\
\left(b^{T}, c^{T}\right)^{T} \in \mathbb{R}^{p} \times \mathbb{R}^{q}
\end{array}
$$

This is the situation one will have when testing the validity of a phase-based GNSS model in which the ambiguities are considered to be integer. The additional term $C c$, with matrix $C$ known and vector $c$ unknown, models under the alternative hypothesis the supposed modeling errors (e.g. outliers, slips, instrumental biases, etc). The standard theory of hypothesis testing would be applicable in case the vector $a$ is real valued. However, this theory is not applicable in our case, since $a$ is known to be integer valued (NB: stating that $a$ is known to be integer valued, is of course not the same as stating that $a$ is known).

In order to be able to validate the above integerbased GNSS model, one must be able to answer questions like:

1. What are the appropriate test statistics for testing $H_{0}$ against $H_{a}$ ?

2. How are these test statistics distributed under $H_{0}$ and $H_{a}$ ?

3. What are the appropriate acceptance- and rejection regions?

However, no rigorous answers to these questions are yet available. Here we will introduce a first solution, albeit restricted to the case of data snooping.

\subsection{Data Snooping for the Integer-Based GNSS Model}

Data snooping implies that one screens the observations one at a time for potential outliers. Now, let $y_{0}$ be the observation to be tested and let $\check{e}_{0}$ be its predicted residual, i.e. the difference between $y_{0}$ and the integerbased prediction of $y_{0}$ based on all other observations (thus with $y_{0}$ excluded). The probability density function (PDF) of this predicted residual, denoted as $f_{\check{e}_{0}}(x)$, has been derived in (Teunissen, 2007). Hence, one can now test whether or not it is likely that the observed $\check{e}_{0}$ is a sample from the distribution $f_{\check{e}_{0}}(x)$. If it is, one accepts the observation; if it is not, one rejects the observation. But before one can execute the test, the question of determining the acceptance and rejection regions should be answered. Answering this question is made difficult by the multimodality of $f_{\check{e}_{0}}(x)$. Let $\Psi \subset \mathbb{R}$ be the acceptance region with coverage probability $P\left[\check{e}_{0} \in \Psi\right]=1-\alpha$. Thus the test leads to rejection if $\check{e}_{0} \notin \Psi$. Since we want the rejection to be rare when the underlying model is correct, the false alarm probability $\alpha$ is chosen as a small value. But since there are an infinite number of subsets that can produce this false alarm probability, we still need to determine a way of defining a proper $\Psi$. It seems reasonable to define the optimal subset as the one which has the smallest volume. In that case the probability $1-\alpha$ would be the most concentrated. This acceptance region is given as

$$
\Psi=\left\{x \in \mathbb{R} \mid f_{\check{e}_{0}}(x) \geq \lambda\right\}
$$

where $\lambda$ is chosen so as to satisfy the given probability constraint. Note, due to the multimodality of $f_{\check{e}_{0}}(x)$, that the acceptance region will in general consist of a number of disconnected regions. This is illustrated in Fig. 2.

Due to the multimodality of the PDF, it is a nontrivial task to decide for a certain given $\alpha$ whether or not an observed sample of $\check{e}_{0}$ leads to rejection. The complication resides in the direct determination of $\lambda$ from $\alpha$. This complication can be avoided, however, if we make use of a Monte Carlo based approach. The computational steps for executing the test are then as follows. Given the observed sample of the prediction error, say $\check{e}_{0}^{*}$, one first computes $\lambda^{*}=f_{\check{e}_{0}}\left(\check{e}_{0}^{*}\right)$. This implies that the sample would lie on the boundary of the acceptance region if $\lambda$ would be set equal to $\lambda^{*}$. Hence, this subset is given as $\Psi^{*}=\left\{x \in \mathbb{R} \mid f_{\check{e}_{0}}(x) \geq \lambda^{*}\right\}$. The next step is then to compute the value of $\alpha$ that would correspond with $\lambda^{*}: \alpha^{*}=1-P\left[\check{e}_{0} \in \Psi^{*}\right]$. Here the simulation enters. Let $N$ be the number of times a sample is generated from $f_{\check{e}_{0}}(x)$ and let $N_{i}$ be the number of times a generated sample lies in $\Psi$. Then $\alpha^{*}$ can be approximated as $\alpha^{*}=1-N_{i} / N$. The decision to accept or reject the observed sample is then based on the 

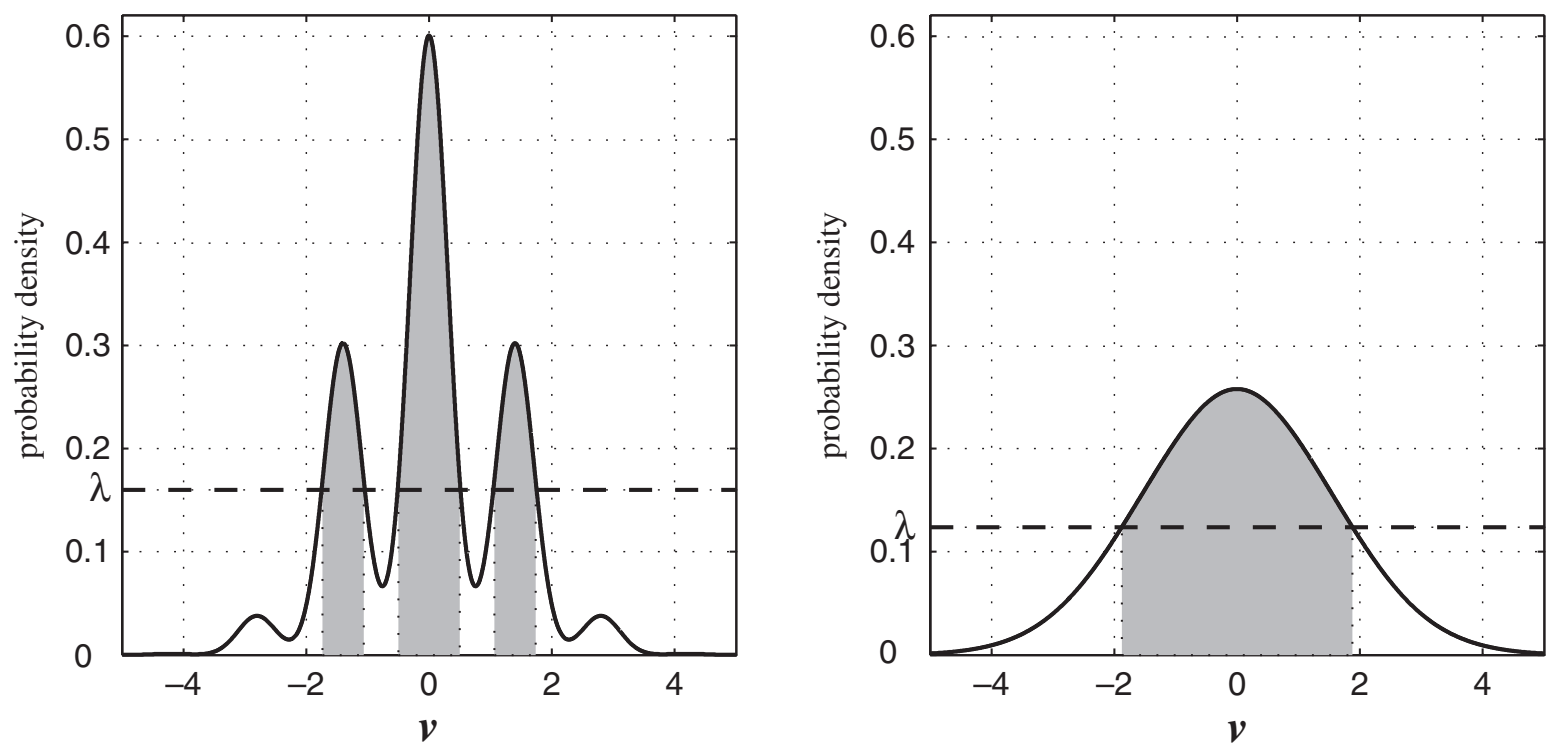

Fig. 2 PDF of the prediction error and corresponding $1-\alpha$ acceptance region (grey areas) for $\sigma=0.31$ (left) and $\sigma=1.00$ (right)

difference between $\alpha$ and $\alpha^{*}$. If $\alpha^{*}<\alpha$, then the test leads to rejection, otherwise it leads to acceptance.

\section{Summary and Conclusions}

In this contribution we addressed three topics for which the theory and/or numerical implementation need further improvements. The three topics are:

1. Ambiguity Acceptance Tests: We have shown that the fixed failure-rate approach should be used when applying the popular ratio-test. Also, the ratio-test can be shown to be suboptimal. For both the optimal test and the fixed failure-rate ratio-test, the numerical difficulty lies, however, in computing the tolerance value $c$ from the user-defined failure-rate. Although methods have been devised to solve this problem, further numerical improvements are required, in particular for real-time applications.

2. Ambiguity Subset Selection: Being able to find the optimal ambiguity subset is of importance for partial AR. In the context of integer boot-strapping an optimal solution is available. This solution, however, is sequentially oriented and not a batch solution. For the latter an optimal solution needs yet to be devised.
3. Integer-Based Model Validation: We have argued that the standard theory of linear model hypothesis testing is not applicable when validating integerbased GNSS models. Hence, a proper theory is still lacking. A first solution, albeit restricted to data snooping, has been presented.

Acknowledgments The research of the first author was done in the framework of his ARC International Linkage Professorial Fellowship, at the Curtin University of Technology, Perth, Australia, with Professor Will Featherstone as his host. This support is greatly acknowledged.

\section{References}

Abidin, H. A. (1993). Computational and geometrical aspects of on-the-fly ambiguity resolution. Ph.D. thesis, Dept. of Surveying Engineering, Techn. Report no. 104, University of New Brunswick, Canada, page 314.

Chen, Y. (1997). An approach to validate the resolved ambiguities in GPS rapid positioning. Proc. of the International Symposium on Kinematic Systems in Geodesy, Geomatics and Navigation, Banff, Canada, pages 301-304.

Euler, H. J. and Schaffrin, B. (1991). On a measure for the discernibility between different ambiguity solutions in the static-kinematic GPS-mode. IAG Symposia no. 107, Kinematic Systems in Geodesy, Surveying, and Remote Sensing, Springer-Verlag, New York, pages 285-295.

Han, S. and Rizos, C. (1996). Validation and rejection criteria for integer least-squares estimation. Survey Review, 33(260):375-382. 
Hofmann-Wellenhoff, B., Lichtenegger, H., and Collins, J. (2001). Global Positioning System: Theory and Practice. Springer-Verlag, Berlin, 5th edition.

Ji, S., Chen, W., Zhao, C., Ding, X., and Chen, Y. (2007). Single epoch ambiguity resolution for Galileo with the CAR and LAMBDA methods. GPS Solutions, DOI 10.1007/s10291007-0057-9.

Joosten, P. and Tiberius, C. C. J. M. (2000). Fixing the ambiguities: are you sure they're right. GPS World, 11(5):46-51.

Koch, K. R. (1999). Parameter Estimation and Hypothesis Testing in Linear Models. Springer Verlag, Berlin, 2nd edition.

Lee, H. K., Wang, J., and Rizos, C. (2005). An integer ambiguity resolution procedure for GPS/pseudolite/INS integration. Journal of Geodesy, 79(4-5): 242-255.

Leick, A. (2003). GPS Satellite Surveying. John Wiley and Sons, New York, 3rd edition.

Misra, P. and Enge, P. (2001). Global Positioning System: Signals, Measurements, and Performance. Ganga-Jamuna Press, Lincoln, MA.

Strang, G. and Borre, K. (1997). Linear Algebra, Geodesy, and GPS. Wellesley-Cambridge Press, Wellesley, MA.

Teunissen, P.J.G. (1997a). A canonical theory for short GPS baselines. Part IV: Precision versus reliability. Journal of Geodesy, 71:513-525.

Teunissen, P.J.G. (1997b). On the GPS widelane and its decorrelation property. Journal of Geodesy, 71(9):577-587.

Teunissen, P.J.G. (1998a). On the integer normal distribution of the GPS ambiguities. Artificial Satellites, 33(2):49-64.

Teunissen, P.J.G. (1998b). Success probability of integer GPS ambiguity rounding and bootstrapping. Journal of Geodesy, 72:606-612.

Teunissen, P.J.G. (1999). An optimality property of the integer least-squares estimator. Journal of Geodesy, 73(11):587-593.

Teunissen, P.J.G. (2001). GNSS ambiguity bootstrapping: Theory and applications. Proc. KIS2001, International Symposium on Kinematic Systems in Geodesy, Geomatics and Navigation, June 5-8, Banff, Canada, pages 246-254.

Teunissen, P.J.G. (2003). Integer aperture GNSS ambiguity resolution. Artificial Satellites, 38(3):79-88.
Teunissen, P.J.G. (2006). Testing Theory, an Introduction. Delft University Press, Delft, 2nd edition.

Teunissen, P.J.G. (2007). Least-squares prediction in linear models with integer unknowns. Journal of Geodesy, DOI:10.1007/s00190-007-0138-0.

Teunissen, P.J.G., Joosten, P., and Tiberius, C. C. J. M. (1999) Geometry-free ambiguity success rates in case of partial fixing. Proc. of National Technical Meeting \& 19th Biennal Guidance Test Symposium ION 1999, San Diego CA, pages 201-207.

Teunissen, P.J.G. and Kleusberg, A. (1998). GPS for Geodesy. Springer, Berlin Heidelberg New York, 2nd edition.

Teunissen, P.J.G. and Verhagen, S. (2004). On the foundation of the popular ratio test for GNSS ambiguity resolution. Proc. of ION GNSS-2004, Long Beach CA, pages 2529-2540.

Teunissen, P.J.G. and Verhagen, S. (2007a). GNSS Phase Ambiguity Validation: A Review. Proc. Space, Aeronautical and Navigational Electronics Symposium SANE2007, The Institute of Electronics, Information and Communication Engineers (IEICE), Japan, 107(2):1-6.

Teunissen, P.J.G. and Verhagen, S. (2007b). The GNSS ratio-test revisited. Submitted to Survey Review.

Verhagen, S. (2005a). On the reliability of integer ambiguity resolution. Navigation, 52(2):99-110.

Verhagen, S. (2005b). The GNSS integer ambiguities: estimation and validation. Ph.D. thesis, Publications on Geodesy, 58, Netherlands Geodetic Commission, Delft

Verhagen, S. and Teunissen, P.J.G. (2006). New global navigation satellite system ambiguity resolution method.compared to existing approaches. Journal of Guidance, Control, and Dynamics, 29(4):981-991.

Vollath, U., Sauer, K., Amarillo, F., and Pereira, J. (2003). Three or four carriers - how many are enough? Proc. of ION GNSS 2003, Portland OR, pages 1470-1477.

Wei, M. and Schwarz, K. P. (1995). Fast ambiguity resolution using an integer nonlinear programming method. Proc. of ION GPS-1995, Palm Springs CA, pages 1101-1110. 